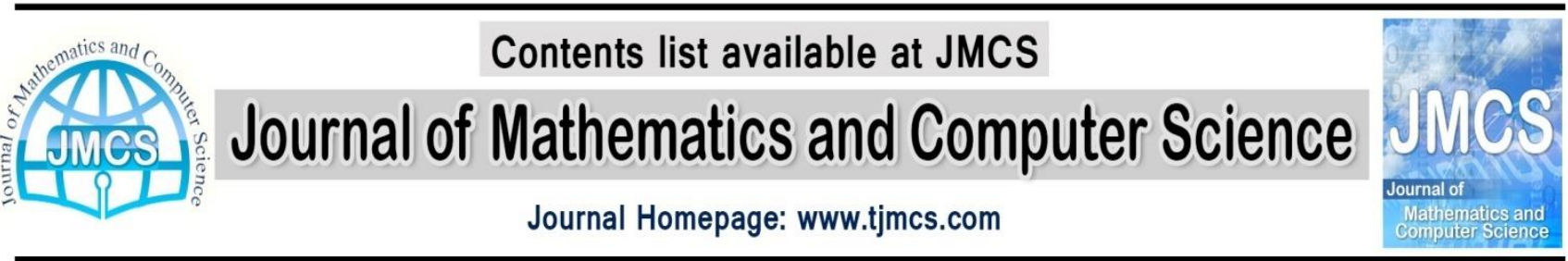

\section{Solving Fractional Partial Differential Equation by Using Wavelet Operational Method}

\author{
A. Neamaty ${ }^{1}$, B. Agheli ${ }^{1}$, R. Darzi ${ }^{2}$ \\ ${ }^{1}$ Department of Mathematics, University of Mazandaran, Babolsar, Iran, \\ namaty@umz.ac.ir; b.agheli@stu.umz.ac.ir \\ ${ }^{2}$ Department of Mathematics, Neka Branch, Islamic Azad University, Neka, Iran \\ darzi@iauneka.ac.ir
}

Article history:

Received May

Accepted June

Available online June

\begin{abstract}
In this paper, we use a method based on the operational matrices to the solution of the fractional partial differential equations. The main approach is based on the operational matrices of the Haar wavelets to obtain the algebraic equations. The fractional derivatives are described in Caputo sense. Some examples are included to demonstrate the validity and applicability of the techniques.
\end{abstract}

Keywords: Operational matrix, Fractional partial differential equation, Haar wavelets, Numerical method.

\section{Introduction}

In recent years, fractional calculus is one of the interest issues that attract many scientists, specially mathematics and engineering sciences. Many natural phenomena can be present by boundary value problems of fractional differential equations. Many authors in different fields such as chemical physics, fluid flows, electrical networks, viscoelasticity, try to modeling of these phenomena by boundary value problems of fractional deferential equations [1-4]. For achieve extra information in fractional calculus, reader can refer to more valuable books that are written by authors [5-9]. Many physical phenomena in areas such as damping laws, diffusion processes, etc, can be modeled with partial differential equations of fractional order. Many authors developed wavelet-based numerical solutions of partial differential equations [10-16].

Useful applications of wavelet operational matrix for numerical solutions of differential equations can be found [17-23]. This issue with characteristic of wavelet functions is motivations for using them to find the solutions of differential equations of fractional order and mostly fractional partial differential equations.

In this paper, we use a method based on the operational matrices to the solution of the fractional partial differential equations. The main approach is based on the operational matrices of the Haar wavelets to obtain the algebraic equations. 


\section{Haar wavelets operational matrixes}

The operational matrix of an orthogonal matrix $\Phi(t), F_{\Phi}$, can be expressed by

$$
F_{\Phi}=\Phi \cdot F_{B} \cdot \Phi^{-1}
$$

where $F_{B}$ is the operational matrix of the block pulse function

$$
F_{B_{m}}=\frac{1}{m}\left[\begin{array}{cccccc}
\frac{1}{2} & 1 & . & . & . & 1 \\
0 & . & . & . & . & . \\
\cdot & . & . & . & . & . \\
\cdot & . & . & . & 1 & . \\
\cdot & . & . & 0 & \frac{1}{2} & 1 \\
0 & . & . & . & 0 & \frac{1}{2}
\end{array}\right] \text {. }
$$

If the transform matrix $\Phi$ is unitary, that is $\Phi^{-1}=\Phi^{T}$ then (2.1) can be rewritten as:

$$
F_{\Phi}=\Phi \cdot F_{B} \cdot \Phi^{T}
$$

The approach is simple and computer oriented, therefore very useful in practice. Wavelets have become an increasingly popular tool in the computational sciences. They have numerous applications in a wide range of areas such as signal analysis, data compression and many others. The Haar wavelets have the following features:(1) highly energy packing; (2) the base functions are consisted of three simple integers, 0 and -1 , and 1 only. The properties are useful in speeding up the computation. So we will use the Haar wavelets and its operational matrix for demonstration throughout this paper. Let us begin by briey reviewing the Haar functions $[24,25]$. The Haar functions are an orthogonal family of switched rectangular waveforms where amplitudes can differ from one function to another. They are defined in the interval $[0,1)$ by

$$
\begin{gathered}
h_{0}(t)=\frac{1}{\sqrt{m}} \\
h_{i}(t)=\frac{1}{\sqrt{m}}\left\{\begin{array}{cc}
2^{\frac{j}{2}} & \frac{k-1}{2^{j}} \leq t \leq \frac{k-\frac{1}{2}}{2^{j}} \\
-2^{\frac{j}{2}} & \frac{k-\frac{1}{2}}{2^{j}} \leq t \leq \frac{k}{2^{j}} \\
0 & \text { otherwise }
\end{array}\right.
\end{gathered}
$$

where $i=1,2, \ldots, m-1, m=2^{r}$ and $r$ is a positive integer. $j$ and $k$ represent the integer decomposition of the index $i$, i.e. $i=2 j+k-1$. Theoretically, this set of functions is complete [24]. In the construction, $h_{0}(t)$ is called the scaling function and $h_{l}(t)$ the mother wavelet. There are two basic operations involved in this set of Haar functions: (1) translation and (2) dilation [25]. Starting from the mother wavelet, $h_{1}(t)$, compression and translation are performed to obtain $h_{2}(t)$ and $h_{3}(t)$ as shown in Fig. 1 . Any function $y(t)$ which is square integrable in the interval $0 \leq t \leq 1$, that is 


$$
\int_{0}^{1} y^{2}(t) d t<\infty
$$

can be expanded into Haar series by

$$
y(t)=c_{0} h_{0}(t)+c_{1} h_{1}(t)+c_{2} h_{2}(t)+\cdots,
$$

where $c_{j}=\int_{0}^{1} y(t) h_{j}(t) d t$.

In usual, the series expansion of (2.6) contains infinite terms for a general smooth function $y(t)$. However, if $y(t)$ is approximated as piecewise constantduring each subinterval, (2.6) will be terminated at finite terms, i.e.

$$
y(t) \approx \sum_{i=0}^{m-1} c_{i} h_{i}(t)
$$

The continuous curve of (2.7) can be written into the discrete form by

$$
y(t) \approx c_{0} h_{0}(t)+c_{1} h_{1}(t)+c_{2} h_{2}(t)+\cdots+c_{m-1} h_{m-1}(t),
$$
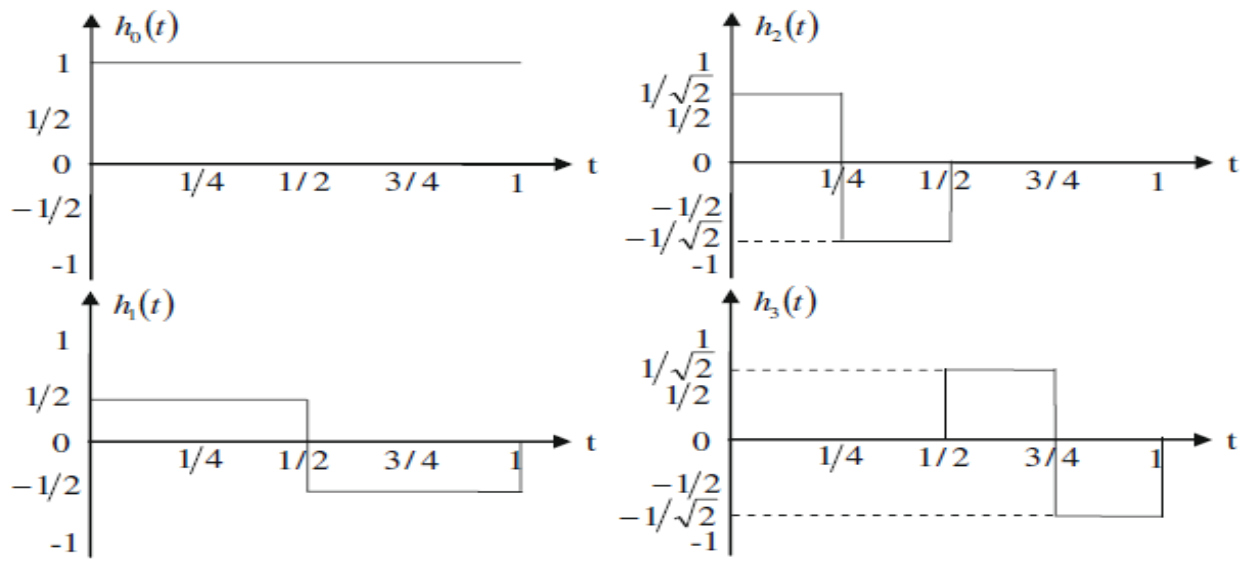

Fig 1: Haar wavelets functions with $m=4$.

where $\mathrm{m}$ is the dimension and usually $m=2^{r}$, is a positive integer. $\vec{y}^{T}=\left[y_{0}, y_{1}, \ldots, y_{m-1}\right]$ is the discrete form of the continuous function $y(t)$, the discrete values yi are obtained by sampling the continuous curve $y(t)$ at a space $\frac{1}{m}$. Similarly,

$\vec{h}_{0}{ }^{T}=\left[h_{0,0}, h_{0,1}, h_{0,2}, \ldots, h_{0, m-1}\right], \vec{h}_{1}^{T}=\left[h_{1,0}, h_{1,1}, h_{1,2}, \ldots, h_{1, m-1}\right], \ldots, \vec{h}_{m-1}{ }^{T}=\left[h_{m-1,0}, h_{m-1,1}, h_{m-1,2}, \ldots, h_{m-1, m-1}\right]$ of the Haar wavelet bases; the discrete values are taken form the continuous curves $h_{0}(t), h_{1}(t), \ldots, h_{m-1}(t)$, respectivly 


$$
H=\left(\begin{array}{c}
\vec{h}_{o}(t) \\
\vec{h}_{1}(t) \\
\ldots \\
\vec{h}_{m-1}(t)
\end{array}\right)=\left(\begin{array}{cccc}
h_{0,0} & h_{0,1} & \ldots & h_{0, m-1} \\
h_{1,0} & h_{1,1} & \ldots & h_{1, m-1} \\
\ldots & \cdot & \ldots & \ldots \\
h_{m-1,0} & h_{m-1,1} & \ldots & h_{m-1, m-1}
\end{array}\right)
$$

Eq.(2.8) is then expressed $\vec{y}^{T}=\vec{c}^{T} . H$, where $\vec{c}=\left[c_{0}, c_{1}, \ldots, c_{m-1}\right]^{T}$ is called the coefficient vector of $\vec{y}$ and it can be calculated from $\vec{c}^{T}=\vec{y}^{T} \cdot H^{-1}$. Similarly, a two-dimensional function $y(x, t)$ which is square integrable in the interval $O<x<1$ and $O<t<1$ can be expanded into Haar series by

$$
y(x, t)=\sum_{i=0}^{m-1} \sum_{j=0}^{m-1} c_{i j} h_{i}(x) h_{j}(t)
$$

where

$$
c_{i j}=\int_{0}^{1} y(x, t) h_{i}(x) d x \int_{0}^{1} y(x, t) h_{i}(t) d t .
$$

Thus, Eq. (2.10) can be written into the discrete form by

$$
Y(x, t)=H^{T}(x) \cdot C \cdot H(t)
$$

where

$$
C=\left(\begin{array}{cccc}
c_{0,0} & c_{0,1} & \ldots & c_{0, m-1} \\
c_{1,0} & c_{1,1} & \ldots & c_{1, m-1} \\
\ldots & \cdot & \ldots & \ldots \\
c_{m-1,0} & c_{m-1,1} & \ldots & c_{m-1, m-1}
\end{array}\right)
$$

is the coefficient matrix of $Y$, and it can be calculated by

$$
C=H \cdot Y \cdot H^{T}
$$

For deriving the operational matrix of Haar wavelets, we let $\Phi=H$ in Eq. (2.3), and obtain

$$
F_{H}=H \cdot F_{B} \cdot H^{T},
$$

where $F_{H}$ is the operational matrix for integration of $\mathrm{H}$. For example, the operational matrix of the Haar wavelet in the case of $m=4$ is given by

$$
F_{H_{4}}=H_{4} \cdot F_{B_{4}} \cdot H^{T}
$$




$$
=\left(\begin{array}{cccc}
\frac{1}{2} & \frac{1}{2} & \frac{1}{2} & \frac{1}{2} \\
\frac{1}{2} & \frac{1}{2} & -\frac{1}{2} & -\frac{1}{2} \\
\frac{1}{\sqrt{2}} & -\frac{1}{\sqrt{2}} & 0 & 0 \\
0 & 0 & \frac{1}{\sqrt{2}} & -\frac{1}{\sqrt{2}}
\end{array}\right)\left(\begin{array}{cccc}
\frac{1}{2} & 1 & 1 & 1 \\
0 & \frac{1}{2} & 1 & 1 \\
0 & 0 & \frac{1}{2} & 1 \\
0 & 0 & 0 & \frac{1}{2}
\end{array}\right)\left(\begin{array}{cccc}
\frac{1}{2} & \frac{1}{2} & \frac{1}{\sqrt{2}} & 0 \\
\frac{1}{2} & \frac{1}{2} & -\frac{1}{\sqrt{2}} & 0 \\
\frac{1}{2} & -\frac{1}{2} & 0 & \frac{1}{\sqrt{2}} \\
\frac{1}{2} & -\frac{1}{2} & 0 & -\frac{1}{\sqrt{2}}
\end{array}\right)=\left(\begin{array}{cccc}
.5 & -.25 & -.0884 & -.0884 \\
.25 & 0 & -.0884 & .0884 \\
.0884 & .0884 & 0 & 0 \\
.0884 & -.0884 & 0 & 0
\end{array}\right) .
$$

For any $m=2^{r}$ where $r$ is a positive integer, we can establish the corresponding operational matrix accordingly.

\section{Numerical evaluation of fractional calculus}

Fractional calculus is a generalization of integration and differentiation to non-integer order. In this section, we use the operational matrix of orthogonal functions to express the fractional derivatives [26]. It consists of the following three steps:

Step 1: Sample the continuous function $f(t)$ into the discrete vector form $\vec{f}=\left[f_{0}, f_{1}, \ldots, f_{m-1}\right]^{T}$, where $\vec{f}$ is a column vector expression.

Step 2: Transfer the vector $\vec{f}$ into the Haar wavelet domain by using the Haar wavelets transform

$$
\vec{f}^{T}=\vec{c}^{T} \cdot H,
$$

where $c^{T}$ is the coeffcient vector and $\mathrm{H}$ is the Haar wavelet matrix.

Step 3: To find the numerical solution of $D_{t}^{\alpha} f=\frac{d^{\alpha} f}{d t^{\alpha}}$, where $\alpha$ is a real number, we use

$$
D_{t}^{\alpha} f=D_{t}^{\alpha}(\vec{c} \cdot H)=\vec{c} D_{t}^{\alpha}(H)
$$

From the definition of the operation matrix, it yields:

$$
D_{t}^{\alpha} H=F_{H}^{-\alpha} \cdot H
$$

where $F_{H}$ is the operational matrix for integration of Haar wavelets. Substituting (3.3) into (3.2) yields:

$$
D_{t}^{\alpha} f=D_{t}^{\alpha}(\vec{c} . H)=\vec{c} F_{H}^{-\alpha} \cdot H
$$

where $F_{H}^{-\alpha}$ is Haar wavelet operational matrix with fractional order and it can be derived from the following equation

$$
F_{H}^{\alpha}=H \cdot F_{B}^{\alpha} \cdot H^{T},
$$

where $F_{B}^{\alpha}$ is the operational matrix of the block pulse function for integration with the order $\alpha$, i.e.

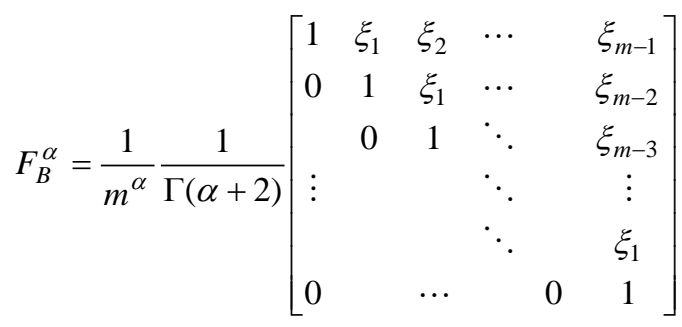

with $\xi_{k}=(k+1)^{\alpha+1}-2 k^{\alpha+1}+(k-1)^{\alpha+1}$. 


\section{Analysis of the method}

The numerical method for the linear fractional partial differential equation is illustrated in this section. This is based on the operational matrices of Haar wavelets.

Obviously, (3.4) can be extended to the case of a function with two variables, the integration order $\alpha$ of $Y(x, t)=H^{T} \cdot C \cdot H(t)$ with respect to variable $t$ yields

$$
\begin{aligned}
I_{t}^{\alpha} Y(x, t) & =I_{t}^{\alpha}\left(H^{T}(x) \cdot C \cdot H(t)\right) \\
& =H^{T}(x) \cdot C \cdot I_{t}^{\alpha} H(t)=H^{T}(x) \cdot C \cdot F_{H}^{\alpha} \cdot H(t)
\end{aligned}
$$

or

$$
I_{t}^{\alpha} Y=H^{T} \cdot C \cdot F_{H}^{\alpha} \cdot H
$$

Similarly, the fractional integration order $\alpha$ of $Y(x, t)$ with respect to variable $\mathrm{x}$ can be expressed as

$$
\begin{aligned}
I_{x}^{\beta} Y(x, t) & =I_{x}^{\beta}\left(H^{T}(x) \cdot C \cdot H(t)\right) \\
& =\left[I_{x}^{\beta} H(x)\right]^{T} \cdot C \cdot H(t)=F_{H}^{\beta} \cdot H^{T}(x) \cdot C \cdot H(t)=H^{T}(x) \cdot\left(F_{H}^{\beta}\right)^{T} \cdot C \cdot H(t)
\end{aligned}
$$

or

$$
I_{x}^{\beta} Y=H^{T} \cdot\left(F_{H}^{\beta}\right)^{T} \cdot C \cdot H
$$

In general, performing the double integration to the function $Y(x, t)$ with fractional order $\alpha$ to variable $\mathrm{t}$ and fractional order $\beta$ to variable $\mathrm{x}$, we obtain

$$
I_{t}^{\alpha} I_{x}^{\beta} Y=H^{T} \cdot\left(F_{H}^{\beta}\right)^{T} \cdot C \cdot F_{H}^{\alpha} \cdot H
$$

Similarly, we have

$$
I_{x}^{\beta} I_{t}^{\alpha} Y=H^{T} \cdot\left(F_{H}^{\beta}\right)^{T} \cdot C \cdot F_{H}^{\alpha} \cdot H
$$

\section{Applications and results}

Example 1. We consider the following fractional Heat equation

$$
\frac{\partial^{\alpha} u(x, t)}{\partial t^{\alpha}}=\frac{1}{\pi^{2}} \frac{\partial^{2} u(x, t)}{\partial x^{2}}
$$

along with the following initial and boundary conditions

$$
\begin{gathered}
u(0, t)=0, \quad \frac{\partial u}{\partial x}(0, t)=\pi E_{\alpha}\left(-t^{\alpha}\right), \quad t>0 \\
u(0, t)=\sin \pi x, \quad x>0
\end{gathered}
$$

The exact solution of (4.1)-(4.3) is $u(x, t)=\sin \pi x E_{\alpha}\left(-t^{\alpha}\right)$. 
Now, we consider (4.1) with the following conditions

$$
\begin{gathered}
u(0, t)=0, \quad \frac{\partial u}{\partial x}(0, t)=x, \quad t>0 \\
u(0, t)=\pi x, \quad x>0
\end{gathered}
$$

Applying the double Laplace transform to both sides of (5.1), we have

$$
s_{2}^{\alpha}\left[L_{t} L_{x} u(x, t)-s_{2}^{-1} \frac{\pi}{s_{1}^{-1}}\right]=\frac{1}{\pi^{2}}\left[s_{1}^{\alpha} L_{t} L_{x} u(x, t)-\frac{\pi}{s_{2}}\right] .
$$

\section{Hence,}

Thus, we have

$$
\left(s_{2}^{\alpha}-\frac{s_{1}^{2}}{\pi}\right) L_{t} L_{x} u(x, t)=s_{2}^{\alpha-1} \frac{\pi}{s_{1}^{2}}-\frac{1}{\pi s_{2}}=\frac{\pi^{2} s_{2}^{\alpha}-s_{1}^{2}}{\pi s_{1}^{2} s_{2}} .
$$

$$
L_{t} L_{x} u(x, t)=\frac{\pi}{s_{1}^{2}} \cdot \frac{1}{s_{2}}
$$

Consequently, $u(x, t)=\pi x$.

Now, we apply the procedure in previous section for $(5.1),(5.4)$ and (5.5). Operating the RiemannLiouville operator $I_{t}^{\alpha}$ on both sides of Eq.(5.1)

$$
u(x, t)-u(x, 0)=\frac{1}{\pi^{2} \Gamma(\alpha)} \int_{0}^{t}(s-t)^{\alpha-1} u_{x}^{\prime \prime}(x, s) d s=\frac{1}{\pi^{2}} I_{t}^{\alpha} u_{x}^{\prime \prime}(x, t) .
$$

Then, integrating (5.6) with respect to $x$ two times, we get

$$
\int_{0}^{x} \int_{0}^{x} u(x, t) d x d x-\pi \int_{0}^{x} \int_{0}^{x} \int_{0}^{x} d x d x d x=\frac{1}{\pi^{2}}\left(I_{t}^{\alpha} u(x, t)-\pi \int_{0}^{x} I_{t}^{\alpha}(1) d x\right)
$$

Let $u(x, t)=H^{T}(x) . C . H(t)$. Substituting (4.1), (4.2) and (4.4) into (5.7), we obtain

$$
\begin{aligned}
& H^{T}(x) \cdot\left(F_{H}^{2}\right)^{T} \cdot C \cdot H(t)-\pi H^{T}(x) \cdot\left(F_{H}^{3}\right)^{T} \cdot J \cdot H(t) \\
& \quad=\frac{1}{\pi^{2}} H^{T}(x) \cdot C \cdot\left(F_{H}^{\alpha}\right)^{T} \cdot H(t)-\frac{1}{\pi} H^{T}(x) \cdot\left(F_{H}^{1}\right)^{T} \cdot J \cdot F_{H}^{\alpha} \cdot H(t)
\end{aligned}
$$

where

$$
J=H \cdot\left(\begin{array}{cccc}
1 & 1 & \cdots & 1 \\
1 & 1 & \cdots & 1 \\
\vdots & \cdot & \vdots & \vdots \\
1 & 1 & \cdots & 1
\end{array}\right)_{m \times m} \cdot H^{T}
$$

By multiplying $H^{T}(x)$ to the right side and $\mathrm{H}(\mathrm{t})$ to the left side of each term in (5.8), it gives

$$
\left(F_{H}^{2}\right)^{T} \cdot C-\pi\left(F_{H}^{3}\right)^{T} \cdot J=\frac{1}{\pi^{2}} \cdot C \cdot F_{H}^{\alpha}-\frac{1}{\pi}\left(F_{H}^{1}\right)^{T} \cdot J \cdot F_{H}^{\alpha}
$$

If the Haar wavelets with order $\mathrm{m}$ is used, (5.10) can be simplifed as:

$$
\left(F_{H_{m}}^{2}\right)^{T} \cdot C_{m \times m}-\pi\left(F_{H_{m}}^{3}\right)^{T} \cdot J_{m}=\frac{1}{\pi^{2}} C_{m \times m} \cdot F_{H_{m}}^{\alpha}-\frac{1}{\pi}\left(F_{H_{m}}^{1}\right)^{T} \cdot J_{m} \cdot F_{H_{m}}^{\alpha}
$$


Solving the above equation to $m=8$ and $\alpha=\frac{1}{2}$, the matrix form of $u(x, t)$ is given by

$\left[\begin{array}{cccccccc}109.1780 & 116.1585 & 88.4466 & 75.2536 & -24.6560 & -156.6537 & -342.2363 & -586.5817 \\ 860.1673 & 822.8567 & 970.9540 & 1041.566 & 1575.925 & 2281.514 & 2371.886 & 4572.098 \\ 1077.383 & 1136.713 & 901.1135 & 789.2779 & -58.8275 & -1180.506 & -2762.631 & -4857.286 \\ 3851.943 & 3375.387 & 5266.210 & 6171.852 & 13009.15 & 22023.91 & 34617.70 & 51016.62 \\ -10971.47 & -8817.750 & -17360.79 & -21462.56 & -52400.09 & -93148.34 & -1.49910^{5} & -2.234062^{5} \\ 50170.69 & 42248.90 & 73663.42 & 88787.45 & 2.0271383^{5} & 3.526208^{5} & 5.6076830^{5} & 8.2889910^{5} \\ -1.764110^{5} & -1.46910^{5} & -2.63810^{5} & -3.20310^{5} & -7.45010^{5} & -1.30310^{6} & -2.07610^{6} & -3.06710^{6} \\ 6.7301010^{7} & 5.641410^{5} & 9.956010^{5} & 1.204410^{6} & 2.773410^{6} & 4.834110^{6} & 7.678210^{6} & 1.130210^{7}\end{array}\right]$

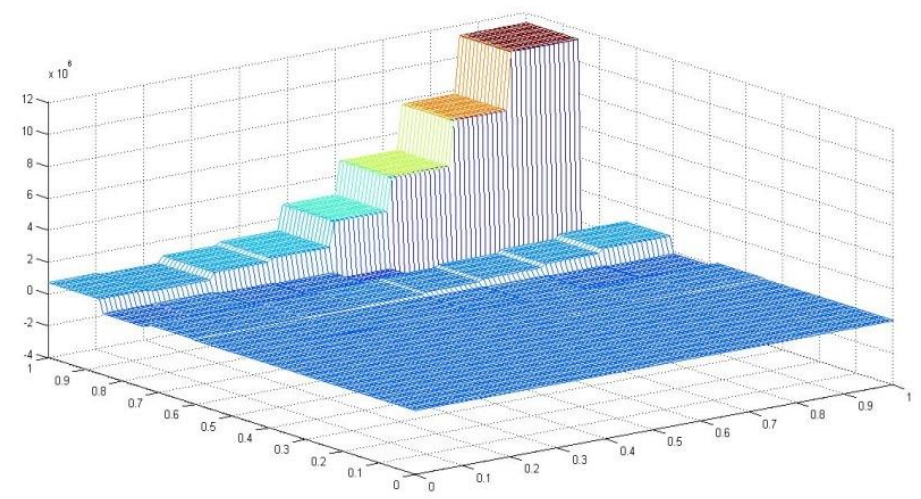

Figure 2: The approximate solution of (4.1)-(4.3) in the case $\alpha=1 / 2$.

Example 2. Consider the following fractional Wave equation

$$
\frac{\partial^{\alpha} u(x, t)}{\partial t^{\alpha}}=k^{2} \frac{\partial^{\beta} u(x, t)}{\partial x^{\beta}}, \quad 1<\alpha<2,1<\beta<2
$$

along with the following initial and boundary conditions

$$
\begin{aligned}
& u(0, t)=0, \quad \frac{\partial u}{\partial x}(0, t)=b_{2}, \quad t>0 \\
& u(x, 0)=0, \quad \frac{\partial u}{\partial x}(x, 0)=b_{1}, \quad t>0
\end{aligned}
$$

where $b_{1}$ and $b_{2}$ are constants.

Operating the Riemann-Liouville operator $I_{t}^{\alpha}$ on both sides of Eq.(5.12) and with boundary conditions (5.14) we have

$$
u(x, t)-\int_{0}^{x} d x-b_{1} \int_{0}^{t} d t=k^{2} I_{t}^{\alpha}\left(\frac{\partial^{\beta} u(x, t)}{\partial x^{\beta}}\right) .
$$


Operating the Riemann-Liouville operator $I_{x}^{\beta}$ on both sides of Eq.(5.15) and with boundary conditions (5.13) we get

$$
I_{x}^{\beta} u(x, t)-I_{x}^{\beta+1}(1)-b_{1} I_{x}^{\beta} I_{t}^{1}(1)=k^{2}\left(I_{t}^{\alpha} u(x, t)-b_{2} I_{t}^{\alpha} I_{x}^{1}(1)\right) .
$$

Let $u(x, t)=H^{T}(x) \cdot C \cdot H(t)$. Substituting (4.1)-(4.4) into (5.16), we obtain

$$
\begin{aligned}
H^{T}(x) \cdot\left(F_{H}^{\beta}\right)^{T} \cdot H(t)-H^{T}(x) \cdot\left(F_{H}^{\beta+1}\right)^{T} \cdot J \cdot H(t)-b_{1} H^{T}(x) \cdot\left(F_{H}^{\beta}\right)^{T} \cdot J \cdot F_{H}^{1} \cdot H(t) \\
\quad=k^{2} H^{T}(x) \cdot C \cdot F_{H}^{\alpha} \cdot H(t)-k^{2} b_{2} H^{T}(x) \cdot\left(F_{H}^{1}\right)^{T} \cdot J \cdot F_{H}^{\alpha} \cdot H(t),
\end{aligned}
$$

where $\mathrm{J}$ is same as (5.9) .

By multiplying $H^{T}(x)$ to the right side and $H(t)$ to the left side of each term in the above equation, for Haar wavelets with order $m$, we have

$$
\begin{aligned}
\left(F_{H_{m}}^{\beta}\right)^{T} \cdot C_{m \times m}-\left(F_{H_{m}}^{\beta+1}\right)^{T} \cdot J_{m}- & b_{1}\left(F_{H_{m}}^{\beta}\right)^{T} \cdot J_{m} \\
& =k^{2} C_{m \times m} \cdot F_{H_{m}}^{\alpha}-K^{2} b_{2}\left(F_{H_{m}}^{1}\right)^{T} \cdot J_{m} \cdot F_{H_{m}}^{\alpha} .
\end{aligned}
$$

Example 3. Consider the following fractional Diffusion-Wave equation

$$
\frac{\partial^{\alpha} u(x, t)}{\partial t^{\alpha}}=\frac{\partial^{2} u(x, t)}{\partial x^{2}}, \quad 1<\alpha \leq 2
$$

subjected to the initial conditions

$$
\begin{aligned}
& u(0, t)=0, \quad \frac{\partial u}{\partial x}(0, t)=b_{2}, \quad t>0 \\
& u(x, 0)=0, \quad \frac{\partial u}{\partial x}(x, 0)=b_{1}, \quad t>0,
\end{aligned}
$$

where $b_{1}$ and $b_{2}$ are constants.

Operating the Riemann-Liouville operator $I_{t}^{\alpha}$ on both sides of Eq.(5.18) and with boundary conditions (5.20) we have

$$
u(x, t)-\int_{0}^{x} d x-b_{1} \int_{0}^{t} d t=I_{t}^{\alpha} u_{x}^{\prime \prime}(x, t) .
$$

Then, integrate (5.21) with respect to $x$ two times, we get

$$
\begin{gathered}
u(x, t)-\int_{0}^{x} d x-\int_{0}^{x} \int_{0}^{x} \int_{0}^{x} d x d x d x-b_{1} \int_{0}^{x} \int_{0}^{x} \int_{0}^{t} d t d x d x \\
=I_{t}^{\alpha} u_{x}(x, t)-I_{t}^{\alpha} \int_{0}^{x} b_{2} d x .
\end{gathered}
$$

Let $u(x, t)=H^{T}(x) \cdot C \cdot H(t)$. Substituting (4.1)-(4.4) into (5.22), we obtain

$$
\begin{aligned}
H^{T}(x) \cdot\left(F_{H}^{2}\right)^{T} \cdot C \cdot H(t)-H^{T}(x) \cdot\left(F_{H}^{3}\right)^{T} \cdot J \cdot H(t)- & b_{1} H^{T}(x) \cdot\left(F_{H}^{2}\right)^{T} \cdot J \cdot F_{H}^{1} \cdot H(t) \\
& =H^{T}(x) \cdot C \cdot F_{H}^{\alpha} \cdot H(t)-b_{2} H^{T}(x) \cdot\left(F_{H}^{1}\right)^{T} \cdot J \cdot F_{H}^{\alpha} \cdot H(t) .
\end{aligned}
$$


By multiplying $H^{T}(x)$ to the right side and $H(t)$ to the left side of each term in the above equation, for Haar wavelets with order $\mathrm{m}$, we have

$$
\begin{aligned}
\left(F_{H_{m}}^{2}\right)^{T} \cdot C_{m \times m}-\left(F_{H_{m}}^{3}\right)^{T} \cdot J_{m}-b_{1}\left(F_{H_{m}}^{2}\right)^{T} \cdot J_{m} \cdot F_{H_{m}}^{1} & \\
& =C_{m \times m} \cdot F_{H_{m}}^{\alpha}-b_{2}\left(F_{H_{m}}^{1}\right)^{T} \cdot J_{m} \cdot F_{H_{m}}^{\alpha}
\end{aligned}
$$

In the case of $\mathrm{m}=8, \alpha=\frac{1}{2}, \mathrm{~b}_{1}=1$ and $b_{2}=2$, we get the numerical solution by solving the equation as below

$$
\left(F_{H_{8}}^{2}\right)^{T} \cdot C_{8 \times 8}-\left(F_{H_{8}}^{3}\right)^{T} \cdot J_{8}-b_{1}\left(F_{H_{8}}^{2}\right)^{T} \cdot J_{8} \cdot F_{H_{8}}^{1}=C_{8 \times 8} \cdot F_{H_{8}}^{\alpha}-b_{2}\left(F_{H_{8}}^{1}\right)^{T} \cdot J_{8} \cdot F_{H_{8}}^{\alpha}
$$

See Figure3.

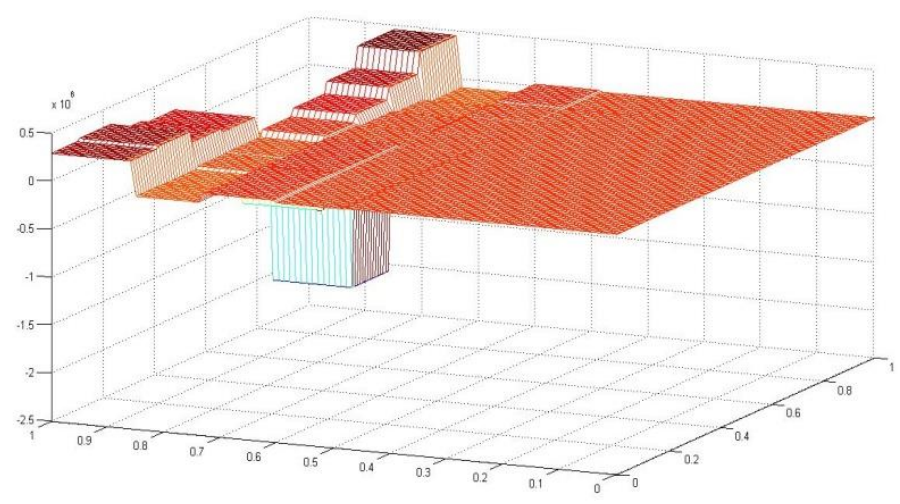

Figure 3: The approximate solution of (4.1)-(4.3) in the case $\alpha=1 / 2, \mathrm{~b}_{1}=1, \mathrm{~b}_{2}=2$.

\section{References}

[1] K.B. Oldham, J. Spanier, The fractional calculus, Academic press, New York and London, (1974).

[2] R. Darzi et al., Sumudu transform method for solving fractional differential equations and fractional Diffusion-Wave equation, tjmcs 6 (2013) 79-84.

[3] F.B. Tatom, Solving Nonlinear Fractional Differential Equations by Bernstein Polynomials Operational Matrices, , tjmcs 5 (2012) 185 - 196.

[4] T.F. Nonnenmacher, R. Metzler, On the Riemann-Liouville fractional calculus and some recent applications, Fractals 3 (1995) 557-566.

[5] S.G. Samko, A.A. Kilbas, O.I. Marichev, fractional integral and derivatives (theory and application), Gordon and Breach, Switzerland, (1993).

[6] A.A. Kilbas, H.M. Srivastava, J.J. Trujillo, Theory and application of fractional differential equations, Elsevier B.V, Netherlands (2006).

[7] K. S. Miller, B. Ross, An introduction to the fractional calculus and fractional differential equation, John Wiley and Sons, New York, (1993).

[8] I. Podlubny, Fractional differential equations, Academic Press, San Diego, CA, (1999). 
[9] V. Lakshmikantham, S. Leela, J. Vasundhara, Theory of fractional dynamic systems, Cambridge Academic Publishers, Cambridge, (2009).

[10] K. Amaratunga et al., Wavelet-Galerkin solutions for one-dimensional partial differential equations, Int. J. Numer. Meth. Eng. 37 (1994) 27052716.

[11] A. Avudainayagam, Wavelet-Galerkin method for integro-differential equations, Appl. Numer. Math. 32 (2000) 247254.

[12] M. El-Gamel, A. Zayed, A comparison between the wavelet-Galerkin and the Sinc-Galerkin methods in solving nonhomogeneous heat equations, in: Zuhair Nashed, Otmar Scherzer (Eds.), Contemporary Mathematics of the American Mathematical Society, Series, Inverse Problem, Image Analysis, and medical Imaging, 313, AMS, Providence, (2002).

[13] M. El-Gamel, Wavelet algorithm for the numerical solution of nonhomogeneous time dependent problems, Int. J. Di_er. Equat. Appl. 9 (2004) 169185.

[14] R. Glowiniski, W.M. Lawton, M. Ravachol, E. Tenenbaum, Wavelet solutions of linear and nonlinear elliptic, parabolic and hyperbolic problems in one space dimension, Comput. Methods Appl. Sci. Eng. 4 (1990) 55120.

[15] Xue-Zhang Liang, Solving second kind integral equations by Galerkin methods with continuous orthogonal wavelets, J. Comput. Appl. Math. 136 (2001) 146161.

[16] S. Qian, J. Weiss, Wavelets and the numerical solution of boundary value problems, Appl. Math. Lett. 6 (1993) 4752.

[17] C. Chen, C. Hsiao Haar wavelet method for solving lumped and distributed-parameter systems, IEE P-Contr Theor Appl. 144 (1) (1997)8794.

[18] N. Bujurke, C. Salimath, S. Shiralashetti, Numerical solution of stiff systems from nonlinear dynamics using single-term Haar wavelet series, Nonlinear Dyn. 51 (4) (2008) 595605.

[19] H. Karimi, B. Lohmann, P. Maralani, B. Moshiri, A computational method for solving optimal control and parameter estimation of linear systems using Haar wavelets, Int J Comput Math. 81 (9) (2004) 11211132.

[20] M. Pawlak, Z. Hasiewicz, Nonlinear system identifcation by the Haar multiresolution analysis, IEEE Trans Circuits I. 45 (9) (1998) 945961.

[21] C. Hsiao, W.Wang, Optimal control of linear time-varying systems via Haar wavelets, J Optim Theory Appl. 103 (3) (1999) 641655.

[22] H. Karimi, B. Moshiri, B. Lohmann, P.Maralani, Haar wavelet-based approach for optimal control of second-order linear systems in time domain, J Dyn Control Syst. 11 (2) (2005) 237252.

[23] I. Sadek, T. Abualrub, M.Abukhaled, A computational method for solving optimal control of a system of parallel beams using Legendre wavelets, Math Comput Model. 45 (2007) 12531264.

[24] A.N. Akansu, R.A. Haddad, Multiresolution Signal Decomposition: Transform, Subbands and Wavelets, Academic Press Inc. (1981) pp. 6061.

[25] M. Vetterli, J. Kovacevic, Wavelets and Subband Coding, Prentice-Hall, Englewood Cliffs, New Jersey. (1995) pp. 3234.

[26] J.L. Wu, A wavelet operational method for solving fractional partial differential equations numerically, Appl. Math. Comput. 214 (2009) 3140. 